Klein, Esther Dominique; Bremm, Nina

\title{
"It's almost as if I treat the teachers as I want them to treat the students". Caring als Facette von Führung an Schulen in sozial deprivierter Lage
} Zeitschrift für Bildungsforschung 9 (2019) 1, S. 89-108

Quellenangabe/ Reference:

Klein, Esther Dominique; Bremm, Nina: "It's almost as if I treat the teachers as I want them to treat the students". Caring als Facette von Führung an Schulen in sozial deprivierter Lage - In: Zeitschrift für Bildungsforschung 9 (2019) 1, S. 89-108 - URN: urn:nbn:de:0111-pedocs-205280 - DOI: 10.25656/01:20528

https://nbn-resolving.org/urn:nbn:de:0111-pedocs-205280 https://doi.org/10.25656/01:20528

\section{Nutzungsbedingungen}

Gewährt wird ein nicht exklusives, nicht übertragbares, persönliches und beschränktes Recht auf Nutzung dieses Dokuments. Dieses Dokument ist ausschließlich für den persönlichen, nicht-kommerziellen Gebrauch bestimmt. Die Nutzung stellt keine Übertragung des Eigentumsrechts an diesem Dokument dar und gilt vorbehaltlich der folgenden Einschränkungen: Auf sämtlichen Kopien dieses Dokuments müssen alle Urheberrechtshinweise und sonstigen Hinweise auf gesetzlichen Schutz beibehalten werden. Sie dürfen dieses Dokument nicht in irgendeiner Weise abändern, noch dürfen Sie dieses Dokument für öffentliche oder kommerzielle Zwecke vervielfältigen, öffentlich ausstellen, aufführen, vertreiben oder anderweitig nutzen.

Mit der Verwendung dieses Dokuments erkennen Sie die Nutzungsbedingungen an.

\section{Terms of use}

We grant a non-exclusive, non-transferable, individual and limited right to using this document.

This document is solely intended for your personal, non-commercial use. Use of this document does not include any transfer of property rights and it is conditional to the following limitations: All of the copies of this documents must retain all copyright information and other information regarding legal protection. You are not allowed to alter this document in any way, to copy it for public or commercial purposes, to exhibit the document in public, to perform, distribute or otherwise use the document in public.

By using this particular document, you accept the above-stated conditions of use.

\section{Kontakt / Contact:}

\section{peDocs}

DIPF | Leibniz-Institut für Bildungsforschung und Bildungsinformation Informationszentrum (IZ) Bildung

E-Mail: pedocs@dipf.de

Internet: www.pedocs.de

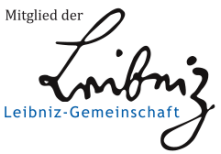




\title{
„It's almost as if I treat the teachers as I want them to treat the students". Caring als Facette von Führung an Schulen in sozial deprivierter Lage
}

\author{
Esther Dominique Klein • Nina Bremm
}

Angenommen: 12. Februar 2019 / Online publiziert: 25. Februar 2019

(C) Springer Fachmedien Wiesbaden GmbH, ein Teil von Springer Nature 2019

Zusammenfassung Die Schulkultur in Schulen in sozial deprivierter Lage ist infolge multipler Misserfolgserfahrungen häufig durch Hilflosigkeit, Abwertungsdynamiken, dysfunktionale Beziehungen und fehlende Verantwortungsübernahme aufseiten der Lehrkräfte geprägt. Insofern besteht eine zentrale Aufgabe im Schulentwicklungsprozess in der „Rekulturierung“ und „Remoralisierung“ dieser Schulen. Der Beitrag stellt vor diesem Hintergrund das Konzept des Caring Leadership als einen innovativen Ansatz vor, mit dem an Schulen in sozial deprivierter Lage eine risikotolerante, sichere Arbeitsumgebung für die Erwachsenen, kollektive Ownership und Verantwortungsübernahme geschaffen werden kann. Anhand von qualitativen Daten aus drei Schulen aus den USA wird in einem explorativen Vorgehen geprüft, welche Formen Caring Leadership annehmen kann und welche Bedeutung dies für den Verlauf von Schulentwicklungsprozessen in den Schulen eröffnen kann. Die Befunde verweisen darauf, dass das Schulleitungshandeln in zwei der Schulen durch Caring geprägt war, was von den schulischen Akteuren als zentraler Faktor für eine erfolgreiche Schulentwicklung und ein positives Schulklima gewertet wird. In der dritten Schule war das Führungsverständnis der Schulleitung dagegen v.a. an einer technokratischen Problemlösung orientiert, weswegen sich die Lehrkräfte nicht ausreichend wertgeschätzt und anerkannt fühlten. Der Beitrag diskutiert mögliche Schlussfolgerungen und benennt weitere Forschungsdesiderata.

\footnotetext{
Anmerkungen Das Projekt „Leadership und Schulentwicklung im Kontext. Eine systematische ländervergleichende Analyse zwischen Nordrhein-Westfalen und Kalifornien“ wurde durch die Deutsche Forschungsgemeinschaft gefördert und an der University of California, Berkeley, durchgeführt. Die Durchführung der Studie wurde durch das Committee for Protection of Human Subjects der University of California, Berkeley, genehmigt.

E. D. Klein $(\triangle) \cdot$ N. Bremm

Fakultät für Bildungswissenschaften, Universität Duisburg-Essen, Universitätsstr. 2, 45141 Essen, Deutschland

E-Mail: dominique.klein@uni-due.de
} 
Schlüsselwörter Caring Leadership $\cdot$ Schulentwicklung $\cdot$ Schulleitung $\cdot$ Schulen in sozial deprivierter Lage $\cdot$ Schulkultur

\title{
"It's almost as if I treat the teachers as I want them to treat the students"-Caring as one element of leadership in schools serving disadvantaged communities
}

\begin{abstract}
The school culture in schools serving disadvantaged communities is often characterized by helplessness, devaluation, dysfunctional relationships, and a lack of responsibility on the part of teachers as a result of multiple experiences of "failure". In this respect, an important step in the improvement process is the "reculturalization" and "remoralization" of these schools. The paper presents the concept of caring leadership as an innovative approach to creating a risk-tolerant, safe work environment for adults, collective ownership and accountability in schools serving disadvantaged communities. Based on qualitative data from three US schools, the paper uses an exploratory approach to examine the forms caring leadership can assume and the significance this can have for school improvement in these schools. Findings indicate that in two of the schools, the site administration used a caring leadership approach, which the school actors considered as a key factor for the success of the schools and for a positive school climate. In the third school, however, the administration's understanding of leadership was mainly positivistic and oriented towards solving problems, and teachers did not feel sufficiently valued and heard. The paper discusses possible conclusions and names further research desiderata.
\end{abstract}

Keywords Caring Leadership $\cdot$ School improvement $\cdot$ School leadership $\cdot$ Schools serving disadvantaged communities $\cdot$ School culture

\section{Einleitung}

Führung gilt als eine der wichtigsten Voraussetzungen für das Gelingen von Schulentwicklung (vgl. Schratz et al. 2016). Bislang greift die Forschung dabei eine positivistische, mechanistische und an Effektivitätskriterien orientierte Perspektive auf Führung von Schulentwicklung auf. Im Vordergrund stehen z. B. Führung von Kooperation und Unterrichtsentwicklung (Harazd und Drossel 2011; Pietsch und Tulowitzki 2017) oder die Verteilung von Führungsaufgaben (Feldhoff und Rolff 2009; Muslic 2017).

Kaum beleuchtet wird dagegen, wie sich Führung auf Organisationskultur und implizite Regelungsstrukturen, Werte und Überzeugungen sowie Beziehungen zwischen schulischen Akteuren auswirkt. So beschreiben auch Wiesner et al. (2015) ein Führungsverständnis, das auf Wohlbefinden, Ko-Konstruktion und Werteorientierung abzielt, als erstrebenswertes Forschungsparadigma, das aktuell jedoch kaum Einzug in die Schulentwicklungsforschung gehalten hat. Besonders deutlich wird dieses Desiderat beim Blick auf Schulen in sozial deprivierter Lage. Auch hier fokussiert die Forschung bislang v. a. Fragen der Effektivität; weniger hinterfragt werden hingegen ungünstige Überzeugungen, Motivationslagen und Einstellungen schuli- 
scher Akteure und wie Führung diese verändern kann. Dieser Beitrag fragt deshalb, wie Führung dazu beitragen kann, in einer Schule mit benachteiligten Standortbedingungen ein positives Schulklima und eine positive Organisationskultur herzustellen und welche Relevanz dies für das Gelingen von Entwicklungsprozessen hat.

Im Folgenden werden zunächst die spezifischen Herausforderungen von Schulen in sozial deprivierter Lage skizziert. Anschließend wird der Ansatz der Caring Leadership (Louis et al. 2016; van de Vyver et al. 2014) beschrieben und es wird hergeleitet, warum Caring Leadership ein sinnvoller Ansatz für die Schulentwicklung in Schulen in sozial deprivierter Lage sein kann. Anhand von qualitativen Daten aus den USA wird anschließend exploriert, welche Formen Caring Leadership annehmen kann und welche Bedeutung dies für den Verlauf von Schulentwicklungsprozessen in Schulen in sozial deprivierter Lage eröffnen kann.

\section{Theoretischer Rahmen}

Schulen in sozial deprivierter Lage beschulen einen überdurchschnittlich hohen Anteil an Schüler/innen aus benachteiligten Herkunftsmilieus. Auch infolge von Passungsproblemen zwischen lebensweltlichen und institutionalisierten Bildungsprozessen sind diese Schulen häufig durch geringere akademische Leistungen sowie ein erhöhtes $\mathrm{Ma} ß$ an Unterrichtsstörungen gekennzeichnet. Daraus ergibt sich vielfach ein erhöhter Entwicklungsbedarf (z.B. Potter et al. 2002). Studien zeigen zudem mehrperspektivische und dialektische Einflussfaktoren auf gelingende Schulentwicklungsarbeit an Schulen in sozial deprivierter Lage, die nicht nur aus den Spezifika einer benachteiligten Schülerzusammensetzung herrühren, sondern auch durch wenig adaptive und eher nachteilige unterrichtliche und organisationale Faktoren geprägt sind (ebd.).

\subsection{Organisationskultur in Schulen in sozial deprivierter Lage}

Diese organisationalen Charakteristika können wiederum durch die individuellen und kollektiven Überzeugungen der schulischen Akteure beeinflusst werden (vgl. Klein 2017), wie Studien aus Deutschland und anderen Ländern aufzeigen: Die Lehrkräfte verorten Ursachen für geringere Leistungen oftmals bei den Schüler/innen und ihren Familien (Bremm und Klein 2017; Nelson und Guerra 2014). Als geteilte Normen und Werte können diese Annahmen in die Organisationskultur (Schein 2004) der Schulen übergehen und Hilflosigkeit, Abwertungsdynamiken, dysfunktionale Beziehungen und fehlende Verantwortungsübernahme erzeugen (z.B. Bremm und Klein 2017; Fölker et al. 2016; Potter et al. 2002; Racherbäumer 2017).

Vor diesem Hintergrund verweist Hemmings (2012) darauf, dass die Organisationskultur der Kern des Problems dysfunktionaler Schulen sei - und zwar insbesondere von Schulen, deren Biografie durch Misserfolgserfahrungen geprägt ist. Häufig sind diese Schulen nicht nur durch fehlende Visionen und ungünstige organisationale Strukturen, sondern auch durch eine „negative“ Organisationskultur und ,widespread resentment, disrespect, apathy, and a pervasive inability [...] to solve problems together" (S. 200) gekennzeichnet. Zudem sind sie oftmals durch 
eine fehlende „Moral“ geprägt, die sich in Pessimismus, gegenseitigem Misstrauen und Aufkündigung des Engagements äußert (ebd., S. 201). In ihrem „4R“-Modell beschreibt Hemmings (2012) vor diesem Hintergrund nicht nur Strategien des „ReEnvisioning“ und des „Restructuring“, sondern auch Strategien der „Reculturation“ und „Remoralization“. Reculturation meint dabei den Aufbau einer positiven Organisationskultur, die es allen Beteiligten ermöglicht, ethisch zu handeln, Verantwortung zu übernehmen, sich mit der Schule zu identifizieren und sich gegenseitig zu unterstützen. Remoralization bedeutet, dass Schulen mit einer pessimistischen, auf Defizite und Hilflosigkeit ausgerichteten Organisationskultur Wege finden, die „Moral“ der Schule wiederaufzubauen. Im Kern einer solchen „Remoralisierung“ steht der Aufbau vertrauensvoller Beziehungen und die Möglichkeit ethischen Handelns (ebd.). Beide Kategorien verweisen insofern nicht auf Strukturen und Prozesse, sondern auf geteilte Normen, Werte und Einstellungen, womit in ihrem Zentrum eine relationale Komponente von Schulentwicklung steht, die über z.B. Kooperationsstrukturen hinausgeht.

Die Schulentwicklungsforschung national wie international hat bislang einen v. a. technokratischen Blick auf die beiden ersten „Rs“ des Modells, d.h. auf Strukturen und Prozesse an Schulen, die optimiert werden müssen (Louis und Murphy 2017). Demgegenüber werden die Relationalität schulischer Arbeit bzw. der Aufbau zwischenmenschlicher Beziehungen und gemeinsamer Werteorientierungen sowie die Gestaltung einer vertrauensvollen Arbeitsumgebung in der Schulentwicklungsforschung bislang höchstens als randständige Faktoren betrachtet und kaum systematisch untersucht. Dies ist umso bemerkenswerter, als dass diverse Forschungsbefunde auf die Relevanz einer vertrauensvollen, wertschätzenden Zusammenarbeit für die Wahrnehmung und Ownership für - und damit auch für den Erfolg von - Entwicklungsstrategien verweisen (z. B. Cosner 2009; Marks et al. 2000; Tschannen-Moran 2009; Brown et al. 2016).

\subsection{Caring Leadership}

In der Folge finden sich in der Literatur bislang kaum systematische Entwicklungsansätze für Schulleitungen, die nicht nur auf Strukturen und Prozesse abzielen, sondern auch auf eine positive Organisationskultur in der Schule. In der schulbezogenen Führungsforschung werden aktuell v.a. Ansätze diskutiert, die im Kern immer auf Organisationsziele und die Entwicklung der Lern- und Arbeitsprozesse und -strukturen an Schulen fokussiert sind. Während einige Ansätze wie der der verteilten Führung (vgl. Spillane et al. 2001) dabei auch die Interessen, Ziele und Wertvorstellungen aller schulischen Akteure in den Blick nehmen oder auf relationale Dimensionen Bezug nehmen (z. B. transformationale Führung, vgl. Bass und Avolio 1994), ist der Aufbau von Beziehungen oder das Wohlbefinden der schulischen Akteure nicht ihr primäres Ziel. Um eine Remoralisierung und Rekulturierung zu erlangen, braucht es insofern eine Führungspraxis, die (auch) den über diese Ziele hinausgehenden Bedürfnissen der Menschen nach Anerkennung und Wohlbefinden einen zentralen Stellenwert einräumt und die Relationalität schulischer Arbeit berücksichtigt (Louis und Murphy 2017). 
In der Organisationssoziologie finden sich verschiedene Führungsansätze, die unter dem Begriff der ethischen Führung firmieren und ein Führungshandeln beschreiben, das durch Altruismus bzw. Fürsorge für andere, aber z. B. auch eine ethische Entscheidungsfindung und Integrität gekennzeichnet ist (vgl. Brown und Treviño 2006; Brown et al. 2005; im Überblick Lang 2014). Ein Ansatz, der im schulischen Kontext v. a. in der nordamerikanischen Literatur diskutiert wird, ist der der Caring Leadership, der von Karen Seashore Louis, Joseph Murphy und Mark Smylie entwickelt wurde. Die Autor/innen übertragen in diesem Ansatz das Prinzip der Fürsorge auf das Führungshandeln in Schulen (Louis et al. 2016; Louis und Murphy 2017; Smylie et al. 2016). Als theoretische Grundlage dient der Fürsorgebegriff von Noddings (2012), in dem Fürsorge als ein tiefes persönliches Interesse an der Person, für die gesorgt wird (attentiveness) und ein Zurückstellen der eigenen Interessen hinter den Interessen der anderen Person (motivational displacement) verstanden wird. Louis et al. (2016) modellieren Caring Leadership vor diesem Hintergrund als ein durch Achtsamkeit gekennzeichnetes Führungshandeln, welches durch echtes Interesse an, Wissen über und Verständnis der Geführten und ihrer Bedürfnisse geprägt und durch die Aussicht auf Erfolg und Wohlergehen für alle schulischen Akteure motiviert wird (S. 319). Das Ziel besteht darin, eine sichere und geschützte, risikotolerante Arbeitsumgebung für die Erwachsenen, kollektive Ownership, Verantwortungsübernahme und eine geringe Fluktuation im Kollegium zu schaffen (Louis et al. 2016) und den in ihr Arbeitenden Wohlbefinden und das Erleben von Selbstwirksamkeit und Sinnhaftigkeit der Arbeit zu ermöglichen (Wiesner et al. 2015). Dem liegt auch die Vermutung zugrunde, dass Caring Leadership über das Arbeitsumfeld und Wohlbefinden der Lehrkräfte dazu beitragen kann, deren fürsorgliches Handeln gegenüber den Schüler/innen zu erhöhen, was wiederum Wohlbefinden, Selbstwirksamkeit und schließlich auch den Bildungserfolg von Schüler/innen beeinflussen kann (Louis et al. 2016).

\subsubsection{Elemente von Caring Leadership}

Caring Leadership umfasst zum einen eine fürsorgliche Einstellung der Führungsperson bzw. Aufmerksamkeit und Verständnis sowie zum anderen ein Führungsverhalten, das den organisationalen Rahmen für eine fürsorgliche Gemeinschaft gestaltet (vgl. auch Smylie et al. 2016). Hierunter subsummieren die Autor/ innen - allerdings nicht trennscharf - Aufmerksamkeit für die Bedürfnisse der Schulmitglieder, Vertrauenswürdigkeit und Zuverlässigkeit, situationsbezogenes Handeln, Authentizität und den Fokus auf die Interessen der Schulmitglieder (Louis et al. 2016). Eine konkrete Operationalisierung dessen, was sich hinter den Begriffen verbirgt, nehmen die Autor/innen gleichwohl nicht vor.

Ein weiterer Ansatz von van de Vyver et al. (2014) nutzt demgegenüber empirische Daten, um - vergleichbar mit dem Ansatz von Louis et al. - zwischen verschiedenen Dimensionen fürsorglicher Führung zu unterscheiden. Die Autor/innen beschreiben zunächst Einstellungen, Kognitionen und Kompetenzen der Führungsperson mit Blick auf fürsorgliches Verhalten. Hierunter fallen beispielsweise emotionale Intelligenz, Interesse an anderen Personen, Empathie, Mitgefühl, Toleranz, Ehrlichkeit und Moral. Eine zweite Dimension umfasst Merkmale der organisationalen 
Umgebung hinsichtlich der Frage, inwiefern diese fürsorgliches Verhalten fördert. Hierzu gehört beispielsweise eine sichere Lernumgebung, das Schulklima und die Verfügbarkeit von Ressourcen. Schließlich beschreiben die Autor/innen Merkmale des fürsorglichen Verhaltens von Führungspersonen. Hierunter fallen etwa Vertrauenswürdigkeit, Empowerment und Anerkennung der geführten Personen, Einbezug der Interessen der geführten Personen, Erreichbarkeit, Konsistenz und Engagement, Unterstützung der geführten Personen sowie Altruismus (van de Vyver et al. 2014).

\subsubsection{Wirkungen von Caring Leadership}

Studien aus der Organisationsforschung zeigen, dass ethische Führung positive Auswirkungen auf Arbeitszufriedenheit, organisationales Commitment, Altruismus, gewissenhaftes Verhalten und Gelassenheit im Umgang mit anderen Mitarbeiter/innen entfalten kann (Sharif und Scandura 2014; Yates 2014). Im Schulbereich lassen sich allerdings bislang nur wenige Studien finden, die sich mit der Fürsorge der Lehrkräfte untereinander bzw. der Schulleitung gegenüber den Lehrkräften befassen (van de Vyver et al. 2014). Einzelne Studien verweisen auf die Bedeutung des Ansatzes für Schulentwicklungsprozesse: die Arbeiten von Louis et al. (2016) etwa lassen darauf schließen, dass Caring Leadership Einfluss auf die Arbeitszufriedenheit und das kollektive Verantwortungsbewusstsein der Lehrkräfte an der Schule nehmen kann und dass eine von den Lehrkräften als durch Fürsorge geprägt wahrgenommene Arbeitsumgebung gepaart mit einem hohen Vertrauen der Schulleitung in die Lehrkräfte mit den Entwicklungsaktivitäten der Lehrkräfte korreliert (Louis und Murphy 2017). Für den deutschsprachigen Raum gibt es Befunde dazu, dass transformationales Führungshandeln (Bass und Avolio 1994), welches Merkmale einer fürsorglichen Führung beinhaltet (Brown et al. 2005), das affektive Commitment der Lehrkräfte gegenüber der Schule erhöhen und Stress und Burnout reduzieren kann (Gerick 2014; Harazd et al. 2012).

Caring Leadership scheint also das Potenzial zu haben, Arbeitszufriedenheit und kollektives Verantwortungsbewusstsein von Lehrkräften positiv zu beeinflussen. Mit seinem Fokus auf die Gestaltung eines auf Risikotoleranz, Empowerment, Ownership und gegenseitige Verantwortung ausgelegten Arbeitsumfeldes ist Caring Leadership insofern vermutlich besonderes dazu geeignet, eine „Rekulturierung“ und „Remoralisierung“ (Hemmings 2012) von Schulen in sozial deprivierter Lage unterstützen zu können. Bislang gibt es aber keine Studien, die diesen Zusammenhang näher beleuchten. Zwar berichtet etwa Walls (2017) in Fallstudien aus zwei Schulen in sozial deprivierter Lage aus den USA einen hohen Zusammenhang zwischen organisationalen Formen des Caring und der Fähigkeit der Lehrkräfte, auf die individuellen Bedürfnisse ihrer Schüler/innen einzugehen und hohe Leistungserwartungen zu etablieren (vgl. auch Walls et al. 2018); allerdings stehen in dieser Studie die Beziehungen zwischen Lehrkräften und Schüler/innen im Fokus. 


\section{Forschungsdesign}

Studien, in denen eine an Fürsorge für die Mitglieder der Schule orientierte Handlungspraxis von Schulleitungen an Schulen in sozial deprivierter Lage systematisch untersucht und zu organisationalen Entwicklungsprozessen in Verbindung gesetzt werden, liegen bislang nicht vor. Auch wird in Abschn. 2 deutlich, dass die verschiedenen Dimensionen von Caring Leadership bislang nur im Ansatz empirisch abgesichert sind.

\subsection{Ziel und Forschungsfragen}

Der vorliegende Beitrag ist vor diesem Hintergrund als Exploration von Caring Leadership und seinem Potenzial für Schulen in sozialräumlich deprivierter Lage zu verstehen. Er greift das beschriebene Desiderat auf und versucht anhand von drei Schulen in sozial deprivierter Lage die Handlungspraxis von Schulleiter/innen nach Elementen der Caring Leadership zu systematisieren und retrospektiv ihre durch die schulischen Akteure wahrgenommene Bedeutung für organisationskulturelle Merkmale in den Schulen herauszuarbeiten. Dabei sollen folgende Fragen beantwortet werden:

1. Inwiefern lassen sich die verschiedenen theoretisch beschriebenen Dimensionen von Caring Leadership in der Handlungspraxis von Schulleiter/innen an Schulen in sozialräumlich deprivierter Lage beobachten?

2. Welche Bedeutung hat Caring Leadership aus der Sicht der Schulleitungen und der Lehrkräfte retrospektiv und aktuell für die Organisationskultur?

\subsection{Stichprobe}

Der Beitrag greift hierzu auf Daten aus einem durch die Deutsche Forschungsgemeinschaft geförderten Projekt zurück, in dem Rollenwahrnehmungen und Führungsstrategien von Schulleiter/innen an Schulen in sozial deprivierter Lage mit unterschiedlich hohen Entwicklungsbedarfen untersucht wurden. Die Daten stammen aus den USA. Dies hat den Vorteil, dass das Konzept zunächst im gleichen institutionellen Kontext analysiert wird, in dem es theoretisch entstanden ist. Befunde aus der Unternehmensforschung verweisen darauf, dass beispielsweise Manager aus den USA ethisches Führungsverhaltens für wichtiger erachten als Manager aus Deutschland (Martin et al. 2009). Insofern muss bei der Frage, welche Rolle Caring Leadership an Schulen in sozial deprivierter Lage spielen kann, auch berücksichtigt werden, welches Verständnis von Führung innerhalb der jeweiligen spezifischen Rahmenbedingungen angemessen ist. ${ }^{1}$

\footnotetext{
1 Daneben können weitere Merkmale der institutionellen Rahmenbedingungen von Schulleitungshandeln und Schulentwicklungsprozessen einen Einfluss darauf haben, wie Caring Leadership umgesetzt wird und welche Wirkungen es entfalten kann. Diese können an dieser Stelle gleichwohl nicht ausgeführt werden. Für einen vertieften Vergleich der institutionellen Rahmenbedingungen in Deutschland und den USA, vgl. z. B. Mintrop und Klein (2017).
} 
Tab. 1 Demographische Daten der Schüler/innen an den drei Schulen (Anteil in Prozent ${ }^{\mathrm{a}}$ )

\begin{tabular}{llll}
\hline & Schule A & Schule B & Schule C \\
\hline Ethnische Herkunft $^{\mathrm{b}}$ & & & \\
$\quad$ Hispanic/Latino & 75 & 95 & 65 \\
$\quad$ Andere & 25 & 5 & 35 \\
English Learners $^{\mathrm{c}}$ & 15 & 25 & 25 \\
Empfänger von kostenfreiem /-reduziertem & 85 & 90 & 55 \\
Essen & & & \\
\hline
\end{tabular}

Quelle: Amtliche Statistik für das Schuljahr 2015/16

${ }^{a}$ Werte sind auf 5 gerundet

${ }^{b}$ Die ethnische Herkunft wird von jedem Schüler/jeder Schülerin als Selbsteinschätzung bei der Einschulung erhoben. Das California Department of Education versteht unter Hispanic/Latino ,, a person of Cuban, Mexican, Puerto Rican, South or Central American, or other Spanish culture or origin, regardless of race“ (https://www.cde.ca.gov/ds/dc/es/refaq.asp\#q8)

${ }^{c}$ Das California Department of Education definiert English Learners als Schüler/innen, ,for whom there is a report of a primary language other than English [...] and who [...] have been determined to lack the clearly defined English language skills of listening comprehension, speaking, reading, and writing necessary to succeed in the school's regular instructional programs" (https://www.cde.ca.gov/ds/sd/cb/glossary.asp\#el)

Die Studie umfasste zwei Teilstudien, in denen quantitative und qualitative Daten sequentiell verbunden wurden. Im ersten, quantitativen Teil wurden sechs Middle Schools in sozial deprivierter Lage in Kalifornien analysiert; im zweiten Teil wurden Vertiefungsstudien an drei der Schulen durchgeführt. Die berichteten Befunde entstammen ausschließlich den qualitativen Vertiefungsstudien. In Tab. 1 werden zentrale Merkmale der Schülerschaft an den drei Schulen dargestellt.

Der Schulleiter ${ }^{2}$ von Schule A hat diese Position seit zehn Jahren; er war zuvor Lehrer im gleichen Schulbezirk. Die Schule hat zwei stellvertretende Schulleiter. Bevor der Schulleiter die Schule übernahm, hatte diese große Probleme mit Disziplinstörungen. Der Schulleiter berichtet, dass es kaum Regeln für die Zusammenarbeit gab. In den ersten Jahren konzentrierte sich die Schulleitung v. a. darauf, klare Regeln aufzustellen und durchzusetzen. Nach zwei Jahren konnte die Schule einen deutlichen Anstieg der Schülerleistungen verzeichnen; die Schule rangierte bis zum Ende des zentralen Testsystems ${ }^{3}$ unter den besten zehn Prozent von Schulen mit vergleichbarer Ausgangslage.

Der Schulleiter von Schule B bekleidet dieses Amt seit vier Jahren und war zuvor lange Zeit stellvertretender Schulleiter der Schule. Die Schule hat zwei stellvertretende Schulleiter. Auch Schule B wird vor der Übernahme durch den Schulleiter als „chaotisch“ bezeichnet. Der Schulleiter führte ein Programm ein, das auf positive Verhaltensintervention setzte, woraufhin sich die Situation grundlegend verbesserte. Zu Beginn befand sich die Schule mit Blick auf die Schülerleistungen in den untersten zehn Prozent vergleichbarer Schulen; kurz nach der Übernahme durch den Schulleiter wurde das zentrale Testsystem beendet.

\footnotetext{
2 Um die Anonymität zu gewährleisten, wird unabhängig vom Geschlecht die männliche Form genutzt.

3 vgl. http://www.ed-data.k12.ca.us/Pages/K-12-changes.aspx.
} 
Der Schulleiter von Schule C arbeitet seit acht Jahren an der Schule; zuvor arbeitete er in einem anderen Schulbezirk. Die Schule hat einen stellvertretenden Schulleiter sowie eine Person, die den Lehrkräften bei der Unterrichtsentwicklung hilft. Die Schülerzusammensetzung in Schule C ist divers; die Schüler/innen kommen z. T. aus sozial benachteiligten, z. T. aber auch aus sehr wohlhabenden Familien. Die Lehrkräfte beschreiben im Gegensatz zu Schule A und B keine „,chaotischen“ Verhältnisse. Mit Blick auf die Schülerleistungen bewegte sich die Schule bis zum Ende des Testsystems im Mittelfeld vergleichbarer Schulen.

\subsection{Methodisches Vorgehen}

In den Vertiefungsstudien wurden die Schulen jeweils für ein bis zwei Wochen besucht. In dieser Zeit wurden leitfadengestützte problemzentrierte Interviews (Witzel 2000) mit den Schulleitern sowie einem Stellvertreter und mit verschiedenen formalen oder informellen Teacher Leaders geführt (Tab. 2). Die Interviews hatten eine Länge von 30 bis $90 \mathrm{~min}$. Die Interviewleitfäden der Schulleiter enthielten retrospektive Fragen zu den von ihnen genutzten Strategien im Entwicklungsprozess sowie Fragen zu aktuellen Strategien in der Entwicklung der Schule. Die Lehrkräfte wurden zudem zu Veränderungen sowie zu aktuellen Strukturen und Prozessen des professionellen Lernens befragt. Dabei wurde nicht explizit nach fürsorglicher Führung gefragt; insofern wurde diese von den Interviewten auch nur dann benannt, wenn sie tatsächlich als relevant für die eigenen Entwicklungsstrategien wahrgenommen wurde.

Zusätzlich wurden an den Schulen ethnografische Beobachtungen durchgeführt. Hierzu wurden die Schulleiter sowie weitere Akteure der Schulleitung an insgesamt zwei bis drei Tagen über mehrere Stunden begleitet. Das Beobachtete wurde anschließend über Feldnotizen rekonstruiert (Emerson et al. 2011). Für alle Interviews liegen Audioaufnahmen vor; alle Audiodateien wurden transkribiert.

Die Auswertung erfolgte anhand der Qualitativen Inhaltsanalyse im Sinne einer inhaltlichen Strukturierung (Mayring 2003). Das Codesystem wurde induktiv und deduktiv entwickelt. In Anlehnung an die Arbeiten von Louis et al. (2016) und van de Vyver et al. (2014) enthielt das Codesystem zunächst die Codes „Persönlichkeitsmerkmale“, „Handlungspraxis“ und „Organisationskulturelle Merkmale“. Diese enthielten verschiedene Subcodes, die insbesondere an der Ausdifferenzierung von van Vyver et al. (2014) orientiert waren. Die „Persönlichkeitsmerkmale“ sollten nur in den Fremdeinschätzungen der Lehrkräfte kodiert werden (vgl. hierzu Lang 2014). Mit diesem Codesystem wurden zunächst 10\% des Materials kodiert; dabei wurden einzelne Subcodes weiter ausdifferenziert und weitere Subcodes ergänzt. Während des Kodierens wurde deutlich, dass sich der Code „Persönlichkeitsmerkmale“ ohne rekonstruktive Analysemethoden nicht valide kodieren ließ, weswegen das Codesys-

Tab. 2 Anzahl Interviews

\begin{tabular}{lll}
\hline & Lehrkräfte & Schulleitung \\
\hline Schule A & $5+1$ Fokusgruppe & 2 \\
Schule B & 6 & 2 \\
Schule C & 3 & 2 \\
\hline
\end{tabular}


Tab. 3 Codesystem

\begin{tabular}{|c|c|c|}
\hline Code & Kategorie & Subcode \\
\hline \multirow[t]{19}{*}{ Handlungspraxis } & \multirow[t]{5}{*}{ Fürsorge-Standards } & Fürsorge für Schüler/innen \\
\hline & & Fürsorge für Lehrkräfte \\
\hline & & Kommunikation der Standards \\
\hline & & Einhalten der Standards prüfen \\
\hline & & Vorbildverhalten \\
\hline & \multirow[t]{3}{*}{ Empowerment } & Professionalität/Autonomie \\
\hline & & Bestätigung/Aufbau der Person \\
\hline & & Anerkennung/Lob \\
\hline & \multirow[t]{4}{*}{ Lehrkräften eine Stimme geben } & Input einholen \\
\hline & & Abweichende Meinungen \\
\hline & & Initiativen der Lehrkräfte \\
\hline & & Lehrkräften eine Stimme geben \\
\hline & \multirow{5}{*}{$\begin{array}{l}\text { Aufmerksamkeit und persönliche } \\
\text { Beziehungen }\end{array}$} & Zuhören/Eingehen auf Sorgen \\
\hline & & Aufmerksamkeit: beruflich \\
\hline & & Aufmerksamkeit: persönlich \\
\hline & & Nahbarkeit \\
\hline & & Positive Beziehungen \\
\hline & \multirow{2}{*}{$\begin{array}{l}\text { Unterstützung und Umgang mit } \\
\text { Fehlern }\end{array}$} & Umgang mit Fehlern \\
\hline & & Unterstützung \\
\hline \multirow[t]{7}{*}{ Schulkultur } & \multirow[t]{7}{*}{-} & Kooperation/Community \\
\hline & & Isolation \\
\hline & & Fluktuation \\
\hline & & Gemeinsame Verantwortung \\
\hline & & Risikotoleranz \\
\hline & & Vertrauen \\
\hline & & Sicherheit \\
\hline
\end{tabular}

tem auf die beiden Codes „Handlungspraxis“ und „Organisationskulturelle Merkmale“ reduziert wurde. Für den Bericht der Ergebnisse wurden die Subcodes von „Handlungspraxis“ in sechs Kategorien systematisiert (vgl. Tab. 3).

Transkripte und ethnografische Berichte wurden jeweils anhand des gleichen Kategoriensystems parallel in MaxQDA kodiert. Tab. 4 illustriert das Vorgehen exemplarisch anhand zweier Subcodes.

Die Codes wurden anschließend paraphrasiert und zu Fällen zusammengefasst. Da die Interviews die Wahrnehmung der Führungspraxis aus der Perspektive verschiedener Akteure (Beobachter, Schulleitung, Lehrkräfte) mit unterschiedlichen Handlungslogiken beschreiben, wurden die Befunde zunächst für die Akteure einzeln pro Schule zusammengefasst (Hartung-Beck und Muslic 2015). Da die Beobachtungen fast immer das Handeln der (stellvertretenden) Schulleiter bzw. ihre Interaktion mit den anderen Mitgliedern der Schule umfassten, wurden die Codes aus den Beobachtungsprotokollen zum Akteur Schulleitung gezählt. Anschließend wurden die Zusammenfassungen für die Schulen einzeln in jedem der Subcodes gegenübergestellt 


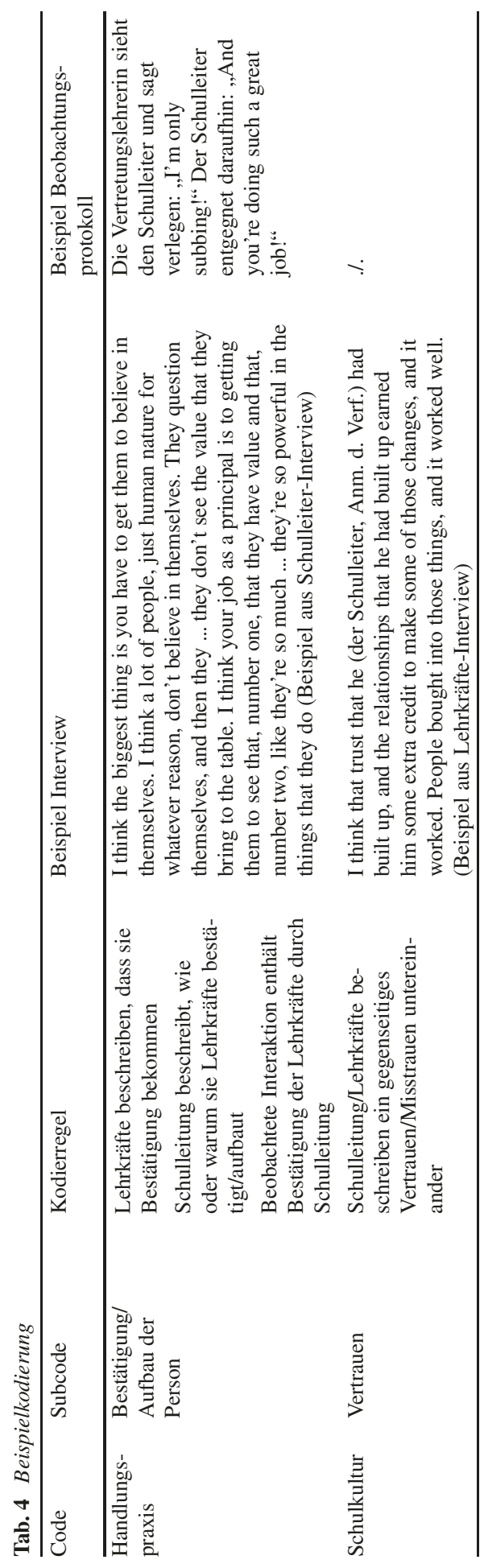


und auf Übereinstimmung bzw. Brüche in der Beschreibung durch die verschiedenen Akteure überprüft.

\section{Ergebnisse}

\subsection{Handlungspraxis}

\subsubsection{Standards für fürsorgliches Handeln}

An den drei Schulen werden Standards für den Umgang der Erwachsenen untereinander - mit Ausnahme von Regelungen für Kooperationssitzungen - nur implizit beschrieben; allerdings wird deutlich, dass die Schulleiter in Schule A und B jeweils einen hohen Anspruch an ihr Verhalten gegenüber den Lehrkräften haben:

I try to remain very consistent because I try to do the same thing that I want that kids have. [...] I try to be consistent, I try to be concise with what my expectations are, and then I try to give them accolades when they deserve them. It's weird. It's almost as if I treat the teachers as I want them to treat the students. (IT; SL-A)

In Schule B zeigt sich dieses Verständnis des Schulleiters v.a. in der Wahrnehmung der eigenen Rolle des „dienenden Schulleiters“ (s. unten).

In Schule C verweist der Schulleiter auf sein Ziel, eine Schule zu schaffen, an der die Lehrkräfte gerne arbeiten; zugleich scheint aber sein Interesse an den Bedürfnissen der Lehrkräfte an bestimmte Bedingungen (z. B. Innovationsbereitschaft) gekoppelt:

I mean I just don't understand the mentality. If you don't like something, fix it. Be part of the solution. If you're not gonna be part of the solution, then go somewhere else. [...] I have no patience for that. (IT; SL-C)

An allen drei Schulen finden sich dagegen vergleichbare Motive der Schulleiter mit Blick auf die Fürsorge für die Schüler/innen. Ihr Ziel besteht im Aufbau von hohen Erwartungen an und persönlichen Beziehungen zu den Schüler/innen, über die das Unterrichtsklima verbessert werden kann. In Schule A und B finden sich diese auch als zentrales Motiv in den Lehrkräfte-Interviews wieder. In Schule C verweisen dagegen nur die Interviews mit dem Schulleiter auf dieses Motiv. Weil der Schulleiter dieses aber nicht klar kommuniziert, fühlen sich einige Lehrkräfte z. T. nicht ausreichend durch die Schulleitung unterstützt:

And a lot of times, that conversation doesn't happen, so the teacher feels that they're not heard. And they have a defiant student in their room, and they're not getting support by administration. And administration feels like you can't kick out all your brown kids and suspend them, but we haven't had that conversation. (IT; L-C) 


\subsubsection{Empowerment}

Mit Blick auf das Empowerment verweisen die Befunde auf divergierende Handlungsstrategien, die durch das grundlegende Führungsverständnis der Schulleiter geprägt sind. Schulleiter A sieht es als eine seiner wichtigsten Aufgaben, den Lehrkräften Selbstwirksamkeit zu vermitteln, damit diese Unterricht verändern und Führungsverantwortung übernehmen können:

They question themselves, and then they don't see the value that they bring to the table. I think your job as a principal is to getting them to see that, number one, that they have value and that, number two, like they're so powerful in the things that they do. (IT; SL-A)

Damit verbunden ist ein hohes Maß an Lob und Anerkennung dessen, was die Lehrkräfte in der Schule leisten. Die Lehrkräfte selbst berichten, wie ,professionals“ behandelt zu werden, was einen positiven Einfluss auf ihre Selbstwirksamkeit sowie die Zusammenarbeit im Kollegium hat.

In Schule B erfolgt Empowerment v.a. über den systematischen Aufbau von Führungsrollen, womit die Hoffnung verbunden ist, auch ein stärkeres Commitment zur Schule und ihrer Vision zu erreichen. Die Lehrkräfte äußern aber z.T. Kritik daran, ob und wie Leistungen anerkannt werden. Zwar lobt der Schulleiter die Lehrkräfte für gute unterrichtliche Leistungen, doch geben auch einzelne Lehrkräfte den Wunsch an, der Schulleiter solle etwas von der Anerkennung, die er für die gute Entwicklung der Schule erhalte, an die Lehrkräfte weitergeben.

Der Schulleiter von Schule C betont an mehreren Stellen die Professionalität der Lehrkräfte, die er versucht, durch Freiräume bei der Gestaltung der Arbeit zu würdigen. Er wünscht sich, dass die Lehrkräfte Risiken eingehen und Führungsverantwortung übernehmen. Von den Lehrkräften wird dieser Ansatz geschätzt; zugleich weisen die Interviews auch darauf hin, dass den Lehrkräften die persönliche Wertschätzung durch den Schulleiter fehlt:

I think just some sort of acknowledgement or something to support that would be a good direction [...]. Build in those structures so that it's embedded within the school culture that we don't just need a leader in order to function. (IT; L06C)

\subsubsection{Lehrkräften eine Stimme geben}

Schulleiter A beschreibt „Zuhören“ als eine zentrale Strategie seiner Führung. Er stößt Gespräche an, um zu erfassen, welche Veränderungen in der Schule gebraucht werden. Zugleich beschreiben die Lehrkräfte den Schulleiter als eine Person, die auch Entscheidungen treffe, die nicht von allen geteilt werden. Zentral dabei ist die Überzeugung der Lehrkräfte, dass der Schulleiter bei seinen Entscheidungen immer fürsorglich handle:

Like even when I'm disagreeing with him I understand he has a reason to make that decision and it's a caring reason, it's an important reason, it's for the good of the order and I respect that. (IT; FG-A) 
In Schule B holen die Schulleitungsmitglieder sich grundsätzlich Input von den Lehrkräften ein; zugleich wird aber auch deutlich, dass der Schulleiter selbst die Entscheidungen trifft und dies auch gegen den Input der Lehrkräfte. Er beschreibt sich selbst als Einzelgänger, der keine Unterstützung benötige und an ein System mit einem Entscheider glaube. Für die interviewten Lehrkräfte ist diese Entschlossenheit einerseits positiv - so wird er als respekteinflößende Person beschrieben, die ihre Vision entschlossen umsetzt. Ein Teil der Lehrkräfte äußert jedoch auch Kritik, da der Schulleiter auf Vorschläge z. T. abweisend reagiere, was auch als entmutigend wahrgenommen wird.

Der Schulleiter von Schule C beschreibt den Einbezug der Lehrkräfte in allen Belangen der Schule als eine der wichtigsten Strategien seines Führungshandelns. In der Schule gibt es im Rahmen von Leadership Teams und Staff Meetings Strukturen, über die Lehrkräfte Sorgen und Wünsche äußern können und bei denen sich der Schulleiter in der Regel zurückhält. Die interviewten Lehrkräfte bestätigen, dass auch abweichende Meinungen nicht nur akzeptiert, sondern sogar gesucht werden. Der inklusive Ansatz des Schulleiters wird von den interviewten Lehrkräften sehr geschätzt und es besteht insgesamt die Wahrnehmung, dass Entscheidungen innerhalb der Schule grundsätzlich nicht unilateral getroffen werden.

\subsubsection{Aufmerksamkeit und persönliche Beziehungen}

In den Schulen A und B berichten die Schulleiter, dass ihr Verständnis für die Sorgen der Lehrkräfte, v. a. mit Blick auf Disziplinprobleme, die Grundlage für das Vertrauen der Lehrkräfte sei und dazu führe, dass diese sich insgesamt stärker engagierten. Schulleiter A etwa interessiert sich für die persönlichen Angelegenheiten der Lehrkräfte und sorgt dafür, dass sie Privates nicht hinter ihre Arbeit stellen. Die Lehrkräfte geben an, dass die Schulleitung den Schüler/innen und Lehrkräften vermittle, dass sie diese ,liebten“, auch wenn es bei Schwierigkeiten Konsequenzen gebe:

Like I said, he's definitely - it's not personal. It's business. But at the time he makes you feel comfortable. He makes you feel as if you're part of the team not even a team - like we're part of a family. (IT; L30-A)

Schulleiter B versucht, für Schüler/innen und Lehrkräfte nahbar zu sein und sich ihnen auch als Menschen zu offenbaren. Er erzählt den Schüler/innen Geschichten aus seinem Leben und ist für die Lehrkräfte jederzeit ansprechbar. In den Interviews wird er als freundlicher, respektvoller Mensch mit großem Interesse an Wohlbefinden und Erfolg der Lehrkräfte beschrieben. Darüber verdeutlicht er auch, dass das berufliche Handeln der Lehrkräfte für ihn eine zentrale Bedeutung hat: Die tägliche Präsenz in den Klassen versteht der Schulleiter als einen der Hauptgründe für die Bereitschaft der Lehrkräfte, sich auf seine Änderungen einzulassen.

Demgegenüber äußern die Lehrkräfte in Schule C das Gefühl, von der Schulleitung nicht ausreichend Aufmerksamkeit v. a. für die grundlegenden Herausforderungen des Lehrerhandelns zu bekommen. Der Schulleiter sei häufig abwehrend und nehme stets eine sachlich-lösungsorientierte Perspektive ein, während viele Lehrkräfte einfach nur gehört und verstanden werden wollten. Aus der Sicht der Lehr- 
kräfte kommt im Führungsverhalten des Schulleiters die persönliche Komponente zu kurz:

I think it would probably be the communication piece, and if we could acknowledge that our profession is both personal and professional [...] and there are gonna be some things that hit very personally, and there are gonna be other things that hit professionally. We need to be able to acknowledge both and be able to say, „Okay, how are we gonna work together for the students and for the teachers?" (IT; L-C)

\subsubsection{Unterstützung und Umgang mit Fehlern}

In den Schulen A und B geben die Lehrkräfte sehr viel Unterstützung z. B. mit Blick auf Classroom Management, Fortbildung und Beziehungen zu den Schüler/ innen an und berichten, jederzeit mit Problemen und Fragen zur Schulleitung gehen zu können. Beide Schulleiter beschreiben sich als Ressource für die Lehrkräfte. Schulleiter A sieht seine Aufgabe v.a. darin, sich um die Dinge zu kümmern, die Lehrkräfte vom Unterrichten abhalten könnten. Schulleiter B beschreibt sich selbst als ,servant leader“, dessen zentrales Ziel das Wachstum jeder einzelnen Person sei:

Job number one, you work for your teachers. You serve them. You serve them. That's your job. Your job isn't to sit in the office, your job isn't to do paperwork and projects; your job is to help your teachers reach those kids. (IT; SL-B)

Die Lehrkräfte beider Schulen berichten auch im Umgang mit Fehlern eine wohlwollende, unterstützende Haltung der Schulleitung. Beide Schulleiter sprechen Schwierigkeiten an, sehen dabei aber immer die Person und versuchen in erster Linie, mit dieser zusammenzuarbeiten, um die Lage zu verbessern.

In Schule $\mathrm{C}$ geht der Großteil der unterrichtlichen Unterstützung nicht von der Schulleitung aus, sondern von der Unterrichtshilfe. Die Schulleitung unterstützt, wenn es um die Besorgung von Materialien oder die Finanzierung von Fortbildung geht, ist aber eher selten in den Klassenräumen, was die Lehrkräfte zwar als Vertrauen in ihre Arbeit interpretieren, zugleich aber auch bemängeln, weil die Schulleitung dadurch wenig Einblick in ihre Arbeit habe und weniger bei Disziplinproblemen unterstütze. Zudem vermerken die Lehrkräfte, dass die Unterstützung durch die Schulleitung v.a. auf Nachfrage durch die Lehrkräfte erfolgt. Die Abwehrhaltung des Schulleiters gibt den Lehrkräften zudem z. T. das Gefühl, nicht genügend unterstützt zu werden und führt aus ihrer Sicht zu einem Misstrauensverhältnis zwischen Kollegium und Schulleitung.

\subsection{Organisationskulturelle Merkmale}

In Schule A und B nutzen die Lehrkräfte häufig das Motiv der Familie, um ihre eigene Schule zu beschreiben. In Schule A verstehen sie sich als ein Team, das gemeinsam das Beste für die Schüler/innen erreichen möchte und diesbezüglich leidenschaftlich agiere; das Schulklima wird als einladend und unterstützend beschrieben und die Schulleitung legt Wert auf eine gute und respektvolle Zusammen- 
arbeit auch bei abweichenden Meinungen. Als einen Grund nennen die Lehrkräfte die insgesamt geringe Fluktuation in der Schule und insofern eine gewachsene und vertrauensvolle Zusammenarbeit. Die Lehrkräfte nehmen sich zudem als autonome und gleichberechtigte „Professionals“ wahr. Deutlich wird zudem das positive Verhältnis zwischen Lehrkräften und Schüler/innen, das in den Interviews vielfach mit dem Begriff „Love“ umschrieben wird.

In Schule B merken die Lehrkräfte ergänzend die Einheit an, die durch den Schulleiter geschaffen wurde:

We're at a point where we're trying to go across curriculums and - Y'know, like history and language arts are working together, and science and language arts are trying to work together. [...] So he's created that kind of an environment to where we're all trying to come together and use what's out there. (IT; L54B)

Es wird allerdings auch deutlich, dass sich das gute Klima v.a. auf den Kern des Kollegiums, der bereits seit vielen Jahren zusammenarbeitet, bezieht. Zugleich sind in den letzten Jahren viele neue Lehrkräfte hinzugekommen, die nicht immer die Philosophie der Schule teilen und vom „Kern“ isoliert sind. Die interviewten Lehrkräfte führen das auf fehlende Team Building-Strategien zurück.

In Schule C bietet sich ein mehrschichtiges Bild. Einerseits geben die interviewten Lehrkräfte zum größten Teil an, innerhalb ihrer Fach- und Jahrgangsteams ein gutes Klima zu haben; andererseits kommt mehrfach - auch aufgrund einer höheren Fluktuation - das Bild von einem segregierten Campus auf, auf dem sich die Lehrkräfte häufig wochenlang nicht sehen und neue Lehrkräfte sich isoliert fühlen. Von der Schulleitung und Lehrkräften mit einem größeren Überblick wird auch die Arbeit in den Teams wegen persönlicher Differenzen bis hin zum Mobbing z.T. als problematisch beschrieben, weswegen mit einzelnen Teams Standards für eine respektvolle Zusammenarbeit erarbeitet werden mussten.

\section{Diskussion}

In den Schulen A und B konnten die Schulleiter erreichen, dass ein Großteil der Lehrkräfte mögliche Defizitperspektiven auf die Schüler/innen abbauen konnten (vgl. vertiefend Klein 2018), sich selbstwirksam fühlen und Verantwortung übernehmen - und so in die Lage versetzt werden, den Schüler/innen gegenüber fürsorglich zu handeln (Louis et al. 2016; Walls 2017). Die Beschreibung der organisationskulturellen Determinanten lässt in beiden Schulen auf ein weitestgehend positives Schulklima schließen, das durch Gemeinschaft, gegenseitiges Vertrauen und eine hohe Risikotoleranz geprägt ist. Mit Blick auf die Herausforderungen von Schulen in sozial deprivierter Lage können die beiden Schulen insofern - unabhängig von den Leistungen der Schüler/innen - als überdurchschnittlich erfolgreich beschrieben werden.

Dabei lassen die vorliegenden Analysen keine Kausalaussagen zu den Gründen des Erfolgs zu; allerdings zeigen die Befunde relativ deutlich, was aus der Sicht der schulischen Akteure ausschlaggebend war. Schulleiter A führt den Erfolg der 
Schule explizit nicht primär auf didaktische oder strukturelle Veränderungen zurück, sondern darauf, dass die Lehrkräfte sich wertgeschätzt und sicher fühlen und deshalb bereit sind, härter zu arbeiten. Schulleiter B sieht den Grund für seinen Erfolg darin, dass die Lehrkräfte durch die Schulleitung Aufmerksamkeit bekommen und sich unterstützt fühlen. Beide Schulleiter sprechen insofern zunächst die psychologische Dimension der Führung von Menschen an, bevor sie auf managerielle oder verwalterische Aufgaben zu sprechen kommen.

Schule C kann in der Retrospektive der interviewten Personen keine vergleichbaren Entwicklungen aufweisen wie Schule A und B, wobei hier auch ein geringerer gefühlter Handlungsdruck besteht als in den anderen beiden Schulen. Die Befunde verweisen zudem darauf, dass Schulleiter C vergleichsweise wenig fürsorgliche Führung betreibt, wobei die Daten keinen Rückschluss auf einen Zusammenhang zwischen den beiden Aspekten zulassen.

In den Lehrkräfte-Interviews spiegelt sich wider, dass das Führungshandeln auch aus Sicht der Lehrkräfte (mindestens) zwei Dimensionen hat, die „managerielle“, bei der die Schulleitung die Funktionalität und Weiterentwicklung der Schule im Blick hat, und die ,zwischenmenschliche“. Diese beiden Dimensionen kommen in den Befunden von Schule A z. B. durch eine klare Trennung - die Lehrkräfte nennen es „Love and Business“ - zum Tragen. In Schule C weisen die Befunde dagegen über das Fehlen von „Love“ auf die Relevanz dieser Dimension hin: Hier trifft ein grundsätzliches Bedürfnis der Lehrkräfte, als Menschen wahrgenommen und bestätigt zu werden, auf eine dazu konträr liegende, problemlösungsorientierte Handlungspraxis des Schulleiters, der zwar die Autonomie- und Selbstverwirklichungsbedürfnisse der Lehrkräfte sieht, Bedürfnisse nach Anerkennung und Wertschätzung aber nicht bedient.

Zudem wird deutlich, dass Caring Leadership mit dem grundsätzlichen Rollenverständnis der drei Schulleiter verbunden ist. Für Schulleiter A und B sind Elemente des Caring zentrale Komponenten der sich selbst zugeschriebenen Führungsrolle. Schulleiter A sieht es als eine seiner wichtigsten Aufgaben an, das Selbstvertrauen von Lehrkräften aufzubauen; Schulleiter B sieht sich als Servant Leader, was konzeptuell eng mit Caring Leadership verknüpft ist (vgl. Lang 2014). Wenngleich sich ihr Führungshandeln nicht vollständig unter diese Rollenverständnisse subsumieren lässt, so machen sie doch deutlich, dass die Schulleiter sich nicht an erster Stelle als Manager oder Qualitätsentwickler sehen.

Demgegenüber beschreibt Schulleiter C als wichtigstes Element seines Handelns den Aufbau einer Schule, in der alle Lehrkräfte Führungsverantwortung übernehmen und Entscheidungen multilateral getroffen werden. In seiner Führungspraxis arbeitet er v.a. an Strukturen und Prozessen. Dabei weisen Passagen sowohl aus dem Schulleiter- als auch aus den Lehrkräfte-Interviews auf Schwierigkeiten dabei hin, Verständnis für die Bedürfnisse von Lehrkräften aufzubringen, die nicht den gleichen problemlösungsorientierten Blick auf Schule einnehmen bzw. Bedürfnisse „unterhalb“ von Autonomie und Selbstverwirklichung haben. Die dadurch entstehenden Missverständnisse führen letztlich in der Wahrnehmung der interviewten Lehrkräfte zu einem Bruch zwischen Schulleitung und Lehrkräften. Insgesamt wird das Schulklima als weniger positiv wahrgenommen als in den anderen beiden Schulen. 


\section{Fazit}

Die Befunde von Louis et al. (2016) sowie Louis und Murphy (2017) zeigen, dass Caring Leadership positiven Einfluss auf die Arbeitszufriedenheit, das kollektive Verantwortungsbewusstsein und die Entwicklungsaktivitäten von Lehrkräften hat. Im Interviewleitfaden, der unserem Material zugrunde liegt, wurde nicht explizit nach Caring Leadership gefragt, so dass hier prinzipiell die Möglichkeit besteht, dass die Ergebnisse verzerrt sind. Gleichwohl wird das Motiv der (fehlenden) Fürsorge durch die Schulleitung in allen drei Schulen sowohl von den Schulleitern selbst, als auch von den interviewten Lehrkräften aufgegriffen. Insgesamt können die Befunde der vorliegenden Studie somit vorsichtig als erster Hinweis dafür gewertet werden, dass Caring Leadership für die Organisationskultur in den Schulen der Studie eine bedeutsame Rolle spielt.

Gleichwohl sind weitere Studien notwendig, die prüfen, inwiefern Caring Leadership in Schulen in sozial deprivierter Lage grundsätzlich Wirkungen auf das Lehrkräftehandeln hat, inwiefern sich diese von Schulen mit anderen Rahmenbedingungen unterscheiden und inwiefern diese Wirkungen schließlich auch von den Schüler/innen wahrgenommen werden. Die vorliegenden Befunde stellen zudem zunächst eine inhaltsanalytische empirische Annäherung an das Caring-Konstrukt dar, die erste Strukturen sichtbar machen kann. Fruchtbar könnte es sein, zusätzlich mit qualitativ-rekonstruktiven Methoden an das Material heranzugehen, um die dem Handeln zugrundeliegenden Orientierungsrahmen der Akteure herauszuarbeiten.

Befunde aus den USA weisen darauf hin, dass Schulleiter/innen in Schulen in sozial deprivierter Lage in geringerem Maße Caring Leadership anwenden als Schulleiter/innen in anderen Schulen (Louis et al. 2016). Hierbei könnte die hohe Fluktuation auf Ebene der Schulleitung in Schulen in sozial deprivierter Lage in den USA eine Rolle spielen (Loeb et al. 2010). Die hier vorgestellten Befunde verdeutlichen, dass dies aber auch mit einem stark am Effektivitätsparadigma orientierten Führungsverständnis von Schulleiter/innen zusammenhängen kann; dieses wiederum könnte an Schulen in sozial deprivierter Lage mit erhöhtem Entwicklungsdruck besonders ausgeprägt sein. Insofern sollten weitere Analysen zur Caring Leadership nicht nur die Wirkungen des Ansatzes auf schulische Prozesse im Blick haben, sondern auch untersuchen, welche Faktoren eine fürsorgliche Führung durch die Schulleitung fördern oder hemmen könnten. Vor diesem Hintergrund lässt sich insgesamt fragen, inwiefern sich institutionelle oder soziokulturelle sowie personelle Rahmenbedingungen auf das fürsorgliche Führungshandeln von Schulleitungen auswirken; dies ist insbesondere insofern relevant, als dass die meisten Befunde zur Caring Leadership - wie die Daten dieser Studie - aus den USA stammen. Hier bedarf es weiterer Studien, welche das Prinzip der fürsorglichen Führung auf einen organisationalen Kontext übertragen, der stärker durch Lehrkräfteautonomie, flache Hierarchien und einen grundsätzlich kooperativeren Schulentwicklungsansatz geprägt ist (vgl. Mintrop und Klein 2017). 


\section{Literatur}

Bass, B. M., \& Avolio, B. J. (1994). Improving organizational effectiveness through Transformational leadership. Thousand Oaks: SAGE.

Bremm, N., \& Klein, E. D. (2017). "No excuses"? Deficit frameworks and responsibility for student success in schools serving disadvantaged communities in Germany. European Conference on Educational Research (ECER), Kopenhagen, Dänemark, 08.2017. Vortrag

Brown, M.E., \& Treviño, L. K. (2006). Ethical leadership. A review and future directions. The Leadership Quarterly, 17(6), 595-616.

Brown, C., Daly, A., \& Liou, Y.-H. (2016). Improving trust, improving schools: Findings from a social network analysis of 43 primary schools in England. Journal of Professional Capital and Community, 1(1), 69-91.

Brown, M.E., Treviño, L. K., \& Harrison, D. A. (2005). Ethical leadership: A social learning perspective for construct development and testing. Organizational Behavior and Human Decision Processes, 97(2), 117-134.

Cosner, S. (2009). Building organizational capacity through trust. Educational Administration Quarterly, 55(1), 248-291.

Emerson, R.M., Fretz, R. I., \& Shaw, L.L. (2011). Writing Ethnographic Fieldnotes (2. Aufl.). Chicago: University of Chicago Press.

Feldhoff, T., \& Rolff, H.-G. (2009). Einfluss von Schulleitungs- und Steuergruppenhandeln. In H.G. Holtappels, K. Klemm \& H.-G. Rolff (Hrsg.), Schulentwicklung durch Gestaltungsautonomie (S. 293-303). Münster: Waxmann.

Fölker, L., Hertel, T., \& Pfaff, N. (2016). Schulische Praxis und Schulentwicklung unter Bedingungen sozialräumlicher Segregation. Befunde einer fallvergleichenden Studie zu zwei Schulen in benachteiligten Stadtteilen. In BMBF (Hrsg.), Steuerung im Bildungssystem (S. 161-176). Berlin: BMBF.

Gerick, J. (2014). Führung und Gesundheit in der Organisation Schule. Zur Wahrnehmung transformationaler Führung und die Bedeutung für die Lehrergesundheit als Schulqualitätsmerkmal. Münster: Waxmann.

Harazd, B., \& Drossel, K. (2011). Formen der Lehrerkooperation und ihre schulischen Bedingungen. Empirische Untersuchung zur kollegialen Zusammenarbeit und Schulleitungshandeln. Empirische Pädagogik, 25(2), 145-160.

Harazd, B., Gieske, M., \& Gerick, J. (2012). Was fördert affektives Commitment von Lehrkräften? Eine Analyse individueller und schulischer (Bedingungs-)Faktoren. Zeitschrift für Bildungsforschung, 2(2), 151-168.

Hartung-Beck, V., \& Muslic, B. (2015). Herausforderungen qualitativer Interviewstudien innerhalb von Organisationen. Methodologische Überlegungen zu einer empirischen Rekonstruktion schulischer Organisationen am Beispiel des Forschungsprojektes „Realisierung testbasierter Schulreform“. Zeitschrift für qualitative Forschung, 16(1), 57-72.

Hemmings, A. (2012). Four Rs for Urban High School Reform. Re-envisioning, Reculturation, Restructuring, and Remoralization. Improving Schools, 15(3), 198-210.

Klein, E. D. (2017). Bedingungen und Formen erfolgreicher Schulentwicklung in Schulen in sozial deprivierter Lage. Eine Expertise im Auftrag der Wübben Stiftung. SHIP Working Paper Reihe, Bd. 01. Essen: Universität Duisburg-Essen. https://doi.org/10.17185/duepublico/44384.

Klein, E. D. (2018). Transformationale Führung und Daten in Schulen in sozial deprivierter Lage. Die Deutsche Schule, 110(1), 27-46.

Lang, R. (2014). Ethische und destruktive Führung. Gute Führung - schlechte Führung. In R. Lang \& I. Rybnikova (Hrsg.), Aktuelle Führungstheorien und-konzepte (S. 313-353). Wiesbaden: Springer VS.

Loeb, S., Kalogrides, D., \& Horng, E.L. (2010). Principal preferences and the uneven distribution of principals across schools. Educational Evaluation and Policy Analysis, 32(2), 205-229.

Louis, K. S., \& Murphy, J. (2017). Trust, caring and organizational learning: The leader's role. Journal of Educational Administration, 55(1), 103-126.

Louis, K.S., Murphy, J., \& Smylie, M. (2016). Caring leadership in schools. Findings from exploratory analyses. Educational Administration Quarterly, 52(2), 310-348.

Marks, H. M., Louis, K. S., \& Printy, S. M. (2000). The capacity for organizational learning. Implications for pedagogical quality and student achievement. In K. Leithwood (Hrsg.), Understanding schools as intelligent systems (S. 239-265). Stamford: JAI Press. 
Martin, G. S., Resick, C. J., Keating, M. A., \& Dickson, M.W. (2009). Ethical leadership across cultures: A comparative analysis of German and US perspectives. Business Ethics: A European Review, 18(2), $127-144$.

Mayring, P. (2003). Qualitative Inhaltsanalyse. Grundlagen und Techniken (8. Aufl.). Weinheim, Basel: Beltz.

Mintrop, R., \& Klein, E. D. (2017). Schulentwicklung in den USA. Nützliches Lehrstück für die deutsche Praxis? In V. Manitius \& P. Dobbelstein (Hrsg.), Schulentwicklungsarbeit in herausfordernden Lagen (S. 63-83). Münster: Waxmann.

Muslic, B. (2017). Kopplungen und Entscheidungen in der Organisation Schule. Organisationsbezogenes Schulleitungshandeln im Kontext von Lernstandserhebungen. Wiesbaden: Springer VS.

Nelson, S.W., \& Guerra, P.L. (2014). Educator beliefs and cultural knowledge. Implications for school improvement efforts. Educational Administration Quarterly, 50(1), 67-95.

Noddings, N. (2012). The caring relation in teaching. Oxford Review of Education, 38(6), 771-781.

Pietsch, M., \& Tulowitzki, P. (2017). Disentangling school leadership and its ties to instructional practice. An empirical comparison of various leadership styles. School Effectiveness and School Improvement, 28(4), 629-649.

Potter, D., Reynolds, D., \& Chapman, C. (2002). School improvement for schools facing challenging circumstances. A review of research and practice. School Leadership \& Management, 22(3), 243-256.

Racherbäumer, K. (2017). Rekonstruktionen zu Bedeutung und Funktionen der Lehrer-Schüler-Beziehung aus Sicht von Lehrerinnen und Lehrern an Schulen in sozial benachteiligter Lage. In V. Manitius \& P. Dobbelstein (Hrsg.), Schulentwicklungsarbeit in herausfordernden Lagen (S. 123-139). Münster: Waxmann.

Schein, E.H. (2004). Organizational culture and leadership (3. Aufl.). San Francisco: Jossey-Bass.

Schratz, M., Wiesner, C., Kemethofer, D., George, A.C., Rauscher, E., \& Krenn, S. (2016). Schulleitung im Wandel: Anforderungen an eine ergebnisorientierte Führungskultur. In M. Bruneforth, F. Eder \& K. Krainer, et al. (Hrsg.), Nationaler Bildungsbericht Österreich 2015 (S. 221-262). Graz: Leykam.

Sharif, M.M., \& Scandura, T.A. (2014). Do perceptions of ethical conduct matter during organizational change? Ethical leadership and employee involvement. Journal of Business Ethics, 124(2), 185-196.

Smylie, M., Murphy, J., \& Louis, K. S. (2016). Caring school leadership: A multi-disciplinary, cross-occupational model. American Journal of Education, 123(1), 1-35.

Spillane, J. P., Halverson, R. \& Diamond, J. B. (2001). Investigating School Leadership Practice. A Distributed Perspective. Educational Researcher, 30(3), 23-28.

Tschannen-Moran, M. (2009). Fostering teacher professionalism in schools. The role of leadership orientation and trust. Educational Administration Quarterly, 55(1), 217-247.

van de Vyver, C.P., van der Westhuizen, P.C., \& Meyer, L.W. (2014). The Caring Leadership Questionnaire (CSLQ). South African Journal of Education, 34(3), 1-7.

Walls, J. (2017). Caring, power, and the emotional and organizational architecture of life in schools. http:// hdl.handle.net/11299/194623 Dissertation. Retrieved from the University of Minnesota Digital Conservancy.

Walls, J., Ryu, J., \& Johnson, J. (2018). A culture of caring and engagement. The case of school leadership at Cedarlane academy. In D. Bickmore \& M. Gawlik (Hrsg.), The charter school principal. Nuanced descriptions of leadership (S. 29-47). Lanham: Rowman \& Littlefield.

Wiesner, C., George, A. C., Kemethofer, D., \& Schratz, M. (2015). School leadership in German speaking countries with an emphasis on Austria: A re-vision. RICERCAZIONE, 7(2), 65-90.

Witzel, A. (2000). Das problemzentrierte Interview. Forum: Qualitative Sozialforschung, 1(1), 1-9. URL: http://www.qualitative-research.net/index.php/fqs/article/view/1132/2519-g1133; Zugriff vom 20.09.2016.

Yates, L.A. (2014). Exploring the relationship of ethical leadership with job satisfaction, organizational commitment, and organizational citizenship behavior. The Journal of Values-Based Leadership, 7(1), 1-15. 